Revue des patrimoines

\title{
Lire / consulter les cartes - reproductions numériques et outils de navigation
}

Reading/consulting maps, digital reproductions and navigational aids

Jean-Luc Arnaud

\section{(2) OpenEdition}

Journals

Édition électronique

URL : http://journals.openedition.org/insitu/22016

DOI : 10.4000/insitu.22016

ISSN : 1630-7305

Éditeur

Ministère de la Culture

Référence électronique

Jean-Luc Arnaud, «Lire / consulter les cartes - reproductions numériques et outils de navigation », In Situ [En ligne], 39 | 2019, mis en ligne le 11 juin 2019, consulté le 11 juillet 2019. URL : http://

journals.openedition.org/insitu/22016 ; DOI : 10.4000/insitu.22016

Ce document a été généré automatiquement le 11 juillet 2019.

\section{cc)}

In Situ Revues des patrimoines est mis à disposition selon les termes de la licence Creative Commons Attribution - Pas d'Utilisation Commerciale - Pas de Modification 4.0 International. 


\section{Lire / consulter les cartes - reproductions numériques et outils de navigation}

Reading/consulting maps, digital reproductions and navigational aids

Jean-Luc Arnaud

1 Cette contribution trouve son origine dans deux expériences: tout d'abord, celle d'un chercheur en histoire urbaine qui exploite la cartographie patrimoniale en la questionnant sur l'organisation de l'espace et les dispositifs qu'elle figure. Cette expérience est assez ancienne pour avoir suivi les multiples progrès des outils numériques, depuis les supports purement analogiques jusqu'au web en passant par l'avènement des photocopieurs en grand format et des possibilités de réduction et d'agrandissement à échelle variable qu'ils proposaient au milieu des années 1980 . Ce savoir, construit dans la durée, a été complété par une expérience plus récente, celle de la mise place d'un site web de documentation cartographique - CartoMundi - Valorisation en ligne du patrimoine cartographique ${ }^{1}$. Enfin, les recherches documentaires afférentes à ces deux expériences m'ont aussi permis d'observer les pratiques des lecteurs dans les cartothèques, à la fois à l'égard des questions qu'ils posent - aux catalogues, aux bibliothécaires et aux documents - et quant à la manière dont ils «enregistrent » les informations.

2 La cartographie se distingue nettement des autres catégories de documents imprimés². Cependant, au sein de la plupart des bibliothèques, les « cartothécaires » sont contraints à exploiter des bases de données structurées pour décrire des livres. Ce n'est pas un hasard si, à la Bibliothèque nationale de France, le département des Cartes et Plans exploite encore un fichier papier pour environ un tiers des documents qu'il conserve. Il s'agit des cartes en série ${ }^{3}$; les outils numériques de catalogage dont il dispose ne permettent pas de prendre en charge les documents graphiques (tableaux d'assemblage) indispensables pour répondre aux questions des lecteurs. Dans ce contexte, les enjeux portés par la numérisation des documents anciens sont multiples. Ils touchent toute leur chaîne de traitement, depuis le catalogage jusqu'à la mise à disposition des lecteurs. 


\section{Un potentiel en devenir}

Sur la base de l'amélioration récente des technologies, de la baisse des coûts d'acquisition des images raster et des politiques de partage de l'information, la numérisation des documents cartographiques imprimés devrait connaitre une forte croissance au cours des prochaines années. Il ne s'agit cependant pas seulement d'effectuer des reproductions ; la multiplicité des traitements envisageables, des formats de publication ou encore des modes de mise à disposition pose de nombreuses questions encore ouvertes. Elles se partagent entre les points de vue des lecteurs et ceux des cartothécaires, en passant par les informaticiens chargés du développement des systèmes et des interfaces.

Pour les lecteurs, les reproductions numériques sont tout d'abord synonymes d'une amélioration de l'accès aux informations cartographiques ${ }^{4}$. Même dans les établissements où les reproductions sont consultables sur place seulement, le temps d'accès aux fichiers est plus rapide que celui aux originaux ${ }^{5}$.

5 Pour les établissements, les reproductions numériques offrent de multiples avantages. Tout d'abord, elles permettent de protéger les collections. La manipulation et la consultation des cartes présentent toujours un risque, d'autant plus important que les documents sont de plus grand format. Les reproductions permettent aussi de délocaliser les collections de manière à les conserver à moindre coût ou bien dans de meilleures conditions qu'à proximité des lieux de consultation. Pour les établissements qui n'ont pas vocation à conserver les documents mais seulement à en faciliter la consultation, ils peuvent se débarrasser des collections reproduites ${ }^{6}$. On ne dispose pas des bilans des expériences récentes en la matière. On peut cependant estimer que les revenus tirés de la vente des documents ont remboursé une forte part des frais de numérisation. Par ailleurs, le coût de conservation et de mise à disposition des reproductions est très inférieur à celui des originaux. Ainsi, que ce soit pour les lecteurs ou pour les établissements, les reproductions numériques présentent un intérêt évident.

6 Au-delà de ces aspects immédiats, les traitements dont les reproductions peuvent être l'objet sont susceptibles de renouveler de manière plus profonde les usages de la cartographie patrimoniale. Depuis la fin des années 2000, les congrès des cartothécaires européens consacrent des ateliers au traitement et à la publication des reproductions numériques ${ }^{7}$. Ils ont donné lieu à la présentation de multiples outils et à autant de débats. De manière générale, les plus innovants ont été proposés par des informaticiens mais la plupart sont restés à l'état de prototypes dans la mesure où les fonctionnalités proposées ne correspondaient ni aux singularités de la documentation cartographique (les cartes ne sont pas seulement des images), ni aux pratiques et aux besoins des lecteurs ou des cartothécaires. Au contraire, les développements portés par des professionnels de la cartographie ont donné des résultats plus durables.

7 Les enjeux portés par la multiplication des reproductions numériques, leurs traitements et leurs usages sont multiples. Nous les avons ici regroupés en cinq chapitres.

\section{Faciliter les consultations}

8 Les reproductions numériques ont pour effet de réduire le temps d'accès à la visualisation des informations cartographiques. En ce sens, elles facilitent la consultation, le nombre 
de documents qu'il est envisageable de consulter au cours de chaque session de travail n'est pas limité par les contraintes d'accès aux magasins et de conservation et enfin, elles permettent de confronter les documents, indépendamment de leur lieu de conservation. L'intérêt des reproductions augmente lorsqu'il est possible d'acquérir les fichiers numériques ou de les consulter en ligne. Depuis une dizaine d'années, le développement des sites web et l'amélioration du débit des réseaux ont donné lieu à la multiplication des reproductions disponibles en ligne.

9 L'expression « lire une carte » est d'un usage courant. Cependant, les cartes - composées en deux dimensions - ne se livrent pas à une investigation séquentielle. Par ailleurs, un texte se déchiffre suivant une seule focale, sa variation ne présente pas d'intérêt, elle ne donne pas lieu à des découvertes supplémentaires. Au contraire, en fonction de la focale, autant d'échelles d'investigation en fait, la consultation d'une carte livre des lots d'informations différents qui peuvent chacun se suffire à eux-mêmes. Ainsi, une carte imprimée sur une feuille de papier résolument plate comporte de facto des données organisées suivant trois dimensions : les deux de l'espace euclidien et la troisième qui correspond à une hiérarchie, celle dont le cartographe a décidé pour rendre compte de l'organisation de l'espace représenté. Cette forme d'organisation des données détermine un mode de consultation particulier; pour saisir une carte dans toutes ses dimensions, il est préférable d'être debout. Dans certaines cartothèques, les tables sont légèrement en pente; elles facilitent la consultation des documents de grand format. Les écrans changent la donne ; il est souvent plus aisé de consulter un détail sur un écran plutôt que sur une table avec une loupe. Par contre, ils sont moins performants que le papier pour les examens d'ensemble. Ils sont trop petits et leur verticalité, qui interdit de tourner autour de la représentation, ne permet pas facilement de varier les angles d'examen.

Face à une carte, le lecteur semble perdu. Plus précisément, il le serait s'il décidait de lire une carte a priori. Or, on fait appel à une carte lorsque l'on a une question à lui poser ; on ne lit pas une carte, on la consulte. Chaque nouvelle question donne lieu à une nouvelle consultation. Par ailleurs, on ne questionne pas ce dont on ignore tout. Ainsi, formuler une question à propos d'une information cartographique suppose de disposer en amont d'une connaissance minimale. Cette connaissance constitue la porte d'entrée nécessaire pour déclencher le questionnement et donc la consultation. Suivant un processus cognitif bien rôdé, on cherche tout d'abord à identifier des points de repère connus, la découverte des autres informations s'organise sur cette base dans un second temps. À partir de ce moment-là, même si les questions ne sont pas explicites, on procède à un questionnement implicite des différences entre ce que l'on connaît et la figuration proposée par la carte. La plus grande part du savoir acquis alors est construite par déduction de ces différences ou des similitudes.

Cette manière d'utiliser les cartes a une forte incidence sur l'exploitation des collections cartographiques en bibliothèque. Qu'il s'agisse de vérifier l'orthographe d'un toponyme, l'existence d'une route ou encore d'un édifice, de nombreuses questions posées aux documents appellent des consultations très courtes. Or, quels que soient les efforts des cartothécaires pour faciliter la consultation de documents, les cartes restent des objets fragiles dont la manipulation prend du temps. Autrement dit, faute de disposer d'un accès rapide aux documents, de très nombreuses questions restent sans réponse. Dans ce contexte, les reproductions numériques et leur publication en ligne fluidifient l'accès à l'information et permettent de multiplier les consultations. Ainsi, il devient envisageable de répondre aux questions qui restaient auparavant sans réponse. En ce sens, la 
dématérialisation des collections cartographiques ne constitue pas seulement un vecteur de promotion des usages: il est probable qu'elle donne lieu à de nouvelles formes d'exploitation, de manière bien plus sensible que pour les textes, avec des applications que l'on ne fait encore qu'entrevoir.

\section{Renouveler le processus de traitement des documents}

12 Au cours des dernières décennies, le monde des cartes a en grande partie échappé à la campagne de rétroconversion des catalogues ${ }^{8}$. Ainsi, le retard accumulé dans la plupart des cartothèques en matière de catalogage risque de devenir un point de blocage pour les reproductions car, a priori, on ne numérise pas un document non catalogué. Faute de catalogues informatisés, les cartothèques se préparent à rater la nouvelle vague de subventions dédiées à la numérisation de masse. La situation économique, qui se traduit par une diminution des moyens humains dans tous les établissements, ne permet pas d'envisager la moindre accélération du rythme de catalogage par des procédés classiques ${ }^{9}$ . Le retard accumulé ne sera pas rattrapé si de nouveaux dispositifs ne sont pas mis en place.

Figure 1

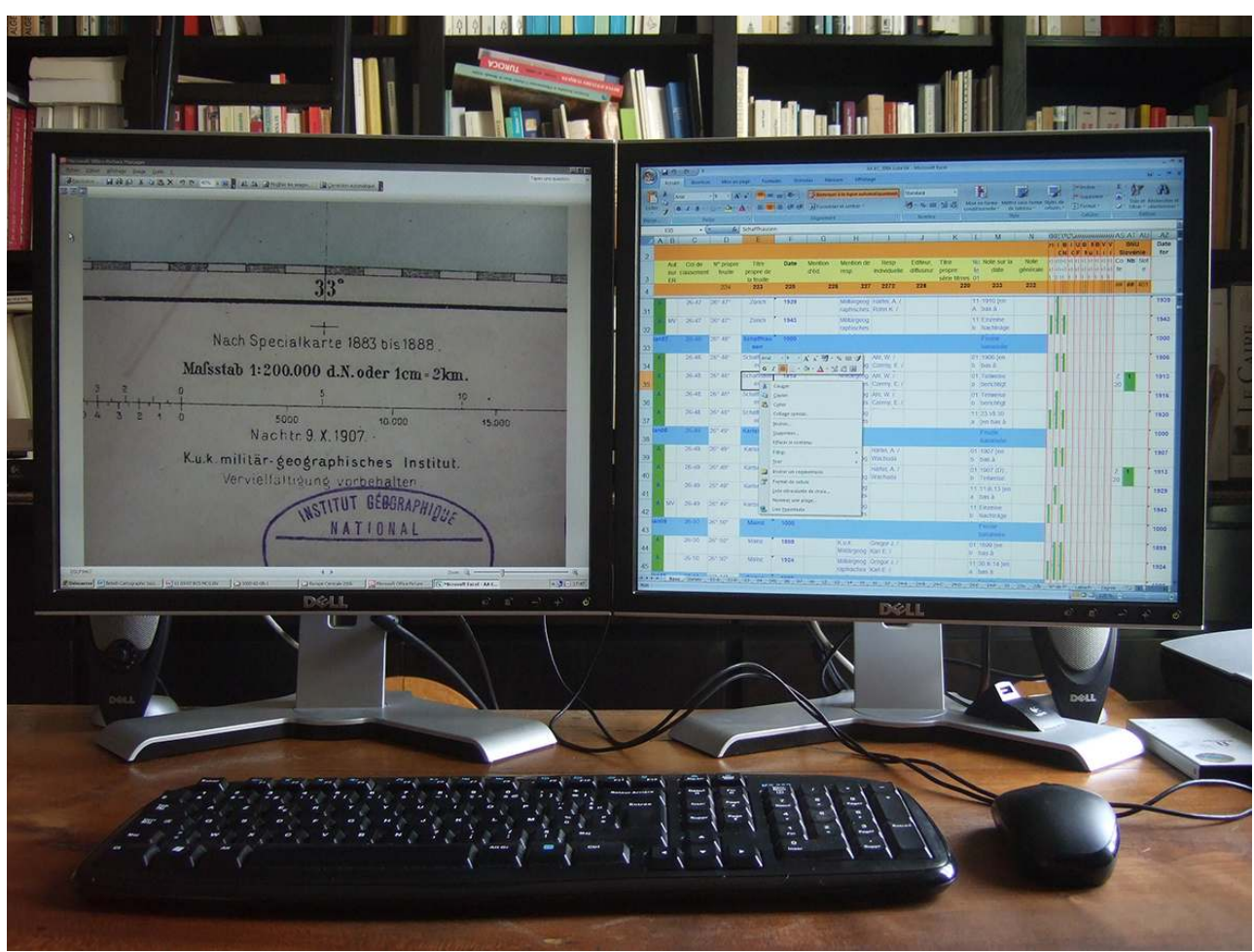

Pour le catalogage des documents (interface de l'écran de droite) la reproduction affichée sur l'écran de gauche facilite la consultation des mentions portées en petits caractères.

(c) Jean-Luc Arnaud.

13 Or, la numérisation offre l'opportunité de repenser la chaîne de traitement des documents (fig. 1). Dans la mesure où il est plus aisé de déchiffrer une mention inscrite en petits caractères sur un écran qu'à l'aide d'une loupe, à plat ventre sur une table, une inversion du processus de traitement des documents, qui débuterait par leur reproduction numérique, semble prometteuse. Des tests effectués dans le cadre de 
CartoMundi sur plusieurs centaines de feuilles à partir de photographies numériques ont montré que le gain de temps peut dépasser $60 \%$ lorsque l'opérateur dispose de deux écrans : un pour le catalogage, un pour la consultation de la reproduction. Le temps gagné est d'autant plus important que les documents sont de plus grand format. Cette méthode présente aussi l'intérêt de déconnecter les travaux de catalogage et la conservation des collections, à la fois dans l'espace et dans le temps; en ce sens, elle simplifie plusieurs aspects logistiques. Les tests ont aussi montré qu'il est envisageable d'exploiter les versions numériques des documents dans le cadre d'un dispositif d'assistance au catalogage. Un tel dispositif associerait des outils de reconnaissance des formes, des symboles et des caractères avec des listes d'autorités, des outils statistiques et une ontologie des relations entre ces éléments ${ }^{10}$. Pour les documents organisés en séries, qui constituent la part la plus abondante de la production cartographique depuis le milieu du $\mathrm{XIX}^{\mathrm{e}}$ siècle, un tel dispositif serait particulièrement performant. Ces documents présentent en effet deux particularités en ce sens. Les métadonnées sont toujours placées en dehors du champ de la représentation cartographique proprement dite, ce qui facilite leur localisation automatique. Par ailleurs, les indications portées sur les feuilles d'une même série présentent des similitudes assez fortes pour en dresser des listes d'autorité et en caractériser la formalisation.

\section{Le géoréférencement en question}

Les documents cartographiques anciens ont été dressés avec des outils dont la précision a connu d'importantes améliorations. Par ailleurs, les multiples systèmes de mesure, méridiens d'origine, modes de projection ou encore échelles de réduction ont donné lieu à une production dont les résultats sont parfois difficiles à comparer terme à terme. Autrement dit, les cartes anciennes se superposent mal entre elles et avec des documents plus récents. Dans ce contexte, on peut se demander s'il ne serait pas souhaitable de transformer les reproductions des documents anciens, suivant des bases géodésiques partagées, pour en faciliter la confrontation.

Les logiciels de déformation des images raster sont déjà anciens. Ils ont été développés au cours des années 1970 pour traiter les images satellites. C'est seulement une vingtaine d'années plus tard que l'on a commencé à les exploiter pour le traitement des documents cartographiques anciens ; il s'agissait alors de systèmes lourds. Au milieu des années 2000, le développement des globes virtuels en ligne a été décisif dans la mesure où ils permettent d'afficher les reproductions déformées de documents anciens sur une image de référence ${ }^{11}$. L'utilisation d'un logiciel de déformation d'images est simple. Sur un écran partagé entre deux fenêtres, une qui affiche la reproduction à transformer, l'autre qui affiche le document de référence, l'opérateur détermine des points de calage sur les deux figurations. À partir de ces indications, la machine calcule la nouvelle position de chaque pixel. Plus le nombre de points est élevé, plus la transformation est précise.

La confrontation des résultats obtenus dans le cadre de plusieurs expériences a montré d'importantes divergences ${ }^{12}$. D'un côté, ceux qui souhaitaient mettre rapidement à disposition de leurs lecteurs des documents géoréférencés en grand nombre mobilisent entre cinq et huit points de calage par feuille, de l'autre, des cartothécaires plus scrupuleux mais moins productifs prétendent que tous les points repérables doivent être exploités. Ils avancent des chiffres de 150 à 200 points de calage par feuille. Si les résultats obtenus sont assez différents, leurs prix de revient le sont aussi. Les débats n'ont pas 
débouché sur une norme ni même sur des prescriptions. Ce n'est pas un hasard; ils ont surtout montré qu'il n'y a pas de bon ni de mauvais géoréférencement mais qu'un même document est susceptible de traitements différents, de précision différente, en fonction des usages que l'on envisage d'en faire. Ainsi par exemple, pour la publication de plusieurs milliers de documents anciens sur le géoportail, l'IGN a limité le nombre des points de calage à trois.

Pour leur part, les historiens préfèrent de manière générale travailler à partir de reproductions brutes dans la mesure où les « erreurs » constituent aussi une source. Elles peuvent permettre d'établir des filiations entre les documents ou encore, en fonction de leur répartition géographique, de définir les lieux d'intérêt de l'auteur ou du commanditaire ${ }^{13}$. Par ailleurs, pour les documents anciens qui figurent des lieux dont les transformations ont été importantes, il n'est pas toujours aisé de repérer des points de calage entre la représentation ancienne et une carte de référence récente. Dans ce cas, il est souhaitable de passer par un ou plusieurs documents intermédiaires, eux-mêmes géoréférencés, et de remonter le temps par étapes successives. Ainsi, le géoréférencement d'un document ancien n'est pas une opération automatisable, l'opérateur est conduit à faire des choix qui ne sont pas sans incidence sur le résultat obtenu. Pour chaque opération, il est souhaitable de conserver en mémoire la position des points de calage et les hésitations qui ont présidé à leur choix pour, éventuellement, affiner le résultat $a$ posteriori. En ce sens, le géoréférencement, trop souvent considéré comme une opération qu'il est envisageable de confier à un technicien, constitue aussi un outil puissant d'investigation des documents anciens. Suivant une procédure hypothético-déductive, par essais et erreurs, le calage d'un document ancien peut être l'occasion de comprendre des dispositions qui, sur la base de l'examen du document brut, ne sont pas interprétables, il peut éclairer la lecture d'une description voire donner lieu à de véritables découvertes.

Dans ce contexte, les grands projets de géoréférencement a priori de collections complètes, tels qu'ils étaient envisagés il y a une dizaine d'années, ont finalement été abandonnés. Par contre, la facilité d'utilisation des outils de géoréférencement a favorisé leur mise à disposition en ligne ${ }^{14}$.

\section{Mosaïque / feuille à feuille}

Dans le cadre du portail de données cartographiques développé par l'IGN pour la France le géoportail -, les premiers documents publiés en ligne ont été tirés de la carte la plus récente ${ }^{15}$. Il ne s'agit pas d'une carte imprimée mais d'une figuration numérique native construite à partir d'une base de données. Dans ce contexte, le partage de la carte en feuilles - comme c'est le cas avec les versions imprimées - ne présente aucun intérêt ; au contraire. Ainsi, pour chaque échelle de figuration, le géoportail propose un continuum cartographique qui couvre l'ensemble du territoire métropolitain.

Pour l'intégration des cartes anciennes, l'IGN a adopté le même principe. Cependant, les originaux sont partagés en plusieurs feuilles (pas moins de 181 pour la carte de Cassini). Ainsi, après avoir été géoréférencée, l'image numérique de chaque feuille a été découpée et assemblée avec les feuilles voisines. Dans la mesure où les feuilles de la carte de Cassini n'ont pratiquement pas été tenues à jour, la mosaïque correspondante ne présente pas de fortes discontinuités entre les feuilles. Le résultat est assez différent pour la minute manuscrite de la carte d'état-major à l'échelle 1:40 000, dont les relevés de terrain se sont 
étalés sur une trentaine d'années - 1830-1860. Pendant cette période, l'organisation du territoire a été touchée par d'importants aménagements. Ainsi, la mosaïque des feuilles présente de multiples décalages qui touchent en particulier les zones urbanisées et les voies de communication. Par ailleurs, cette mosaïque présente un autre problème, elle n'indique pas la date des relevés ou de la rédaction de chaque unité. Ces informations, portées dans les marges des originaux, sont supprimées par le découpage. Les mosaïques trouvent aussi leurs limites avec la multiplicité des éditions de chaque feuille. Ainsi, par exemple, pour la carte de France à l'échelle 1:50 000 dont le tableau d'assemblage est composé de 1100 cases, on compte plus de 3500 feuilles publiées avec d'importants écarts à la moyenne ${ }^{16}$ (fig. 2). La mosaïque présente moins d'un tiers des feuilles de la série, leur sélection a nécessairement comporté une forte part d'arbitraire. Enfin, la mosaïque constitue aussi un témoignage sans indulgence des approximations de l'original, des différences de facture entre les feuilles et de la précision du géoréférencement. Autrement dit, il n'est pas toujours souhaitable de publier une carte sous forme de continuum.

Figure 2

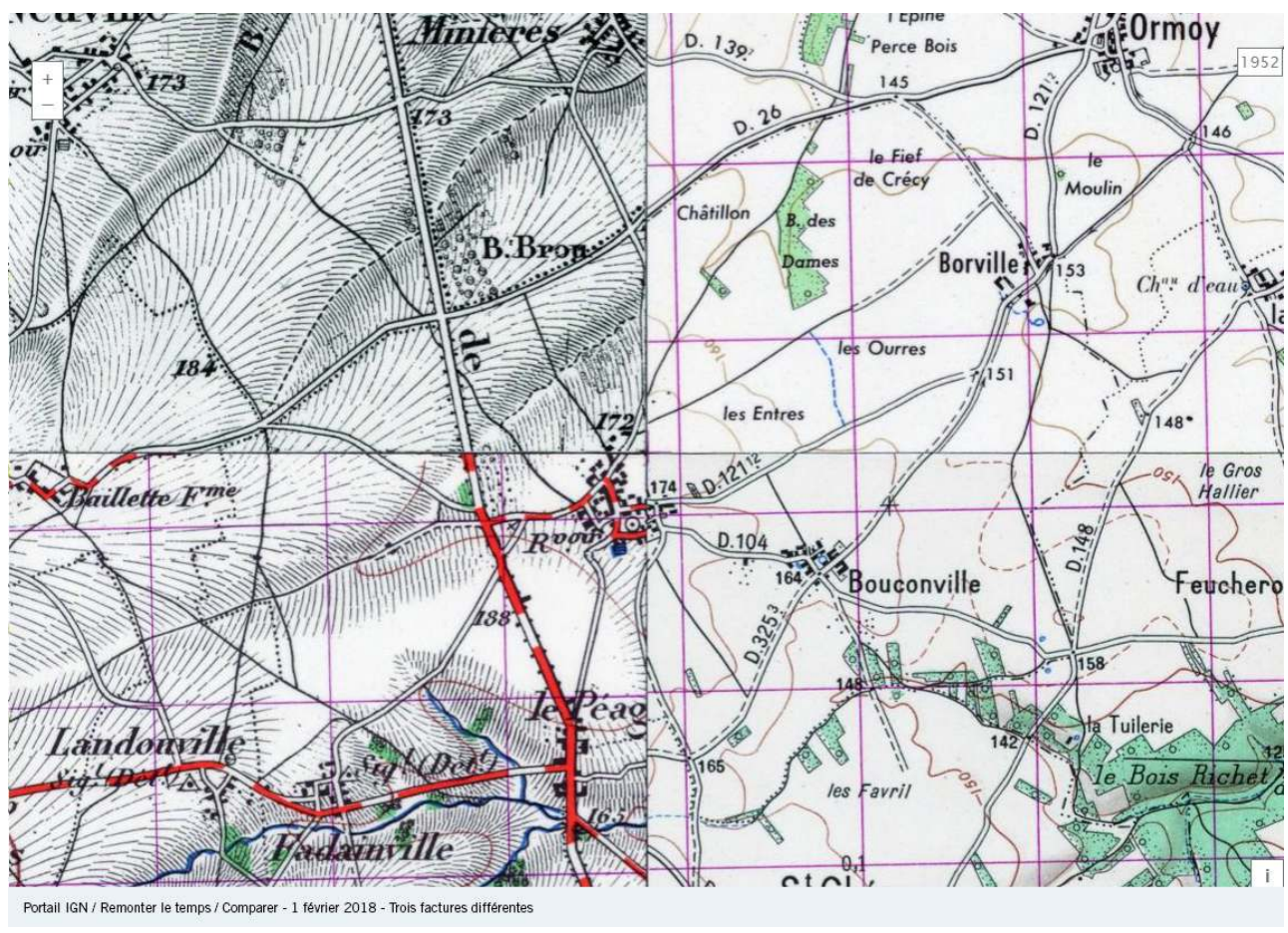

La mosaïque des feuilles de la carte de France à l'échelle 1:50 000 publiée sur le géoportail de l'IGN rassemble des documents de dates et de factures assez diverses pour créer de fortes discontinuités.

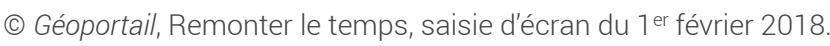

Ce n'est pas un hasard si, pour le géoportail, l'expérience des mosaïques a été limitée à deux cartes seulement : celle de Cassini et la minute manuscrite de la carte d'état-major. Pour sa part, le portail Remonter le temps, aussi développé par l'IGN, présente aussi une mosaïque de la carte topographique au 1:50000. Elle témoigne bien des difficultés présentées par un assemblage de feuilles de date, de qualité et de facture très diverses ${ }^{17}$.

En fait, le résultat du mosaïcage est d'autant plus satisfaisant que la facture des documents traités est plus homogène et que leur période de publication est plus courte. Ainsi, plutôt que dans les grandes couvertures nationales, dont la production s'est 
nécessairement étalée sur plusieurs décennies, le mosaïcage trouve tout son intérêt dans les cartes monographiques partagées en plusieurs feuilles. Il ne s'agit pas de véritables séries mais de documents, composés comme des ensembles, qui ont été découpés en plusieurs unités, soit au moment de l'édition pour faire correspondre le format de la carte avec celui du papier ou de la presse disponible, soit après édition, pour en faciliter la manipulation ${ }^{18}$. Les contributeurs du site web Map Warper ont bien compris cet intérêt. Le site propose plus de trois cents mosaïques dont la plus grande part résulte de l'assemblage de deux à une vingtaine de feuilles ${ }^{19}$.

\section{Nouveaux outils de recherche et de navigation}

La numérisation, en facilitant l'accès à une masse importante de documents, a montré les limites du mode d'organisation des catalogues. La norme de catalogage actuellement en vigueur dans la plupart des cartothèques a été adoptée au cours des années $1970^{20}$. Elle a été conçue par dérivation des principes établis pour les livres, sans tenir compte de la manière dont les lecteurs formulent la plus grande part de leurs requêtes. Ainsi, ces catalogues ne permettent pas de répondre à la question: "Je cherche une carte de tel endroit ». Cette question est primordiale, les critères de sélection par auteur, par date ou encore par échelle interviennent dans un second temps seulement, pour affiner les résultats obtenus en réponse à la question initiale. Cependant, les catalogues sont justement incapables de répondre à cette question dans la mesure où, dans le meilleur des cas, chaque document est qualifié par quelques toponymes seulement alors qu'il figure des centaines de lieux, dont tous les noms ne sont pas inscrits sur la carte (fig. $\mathbf{3}$ ).

Figure 3
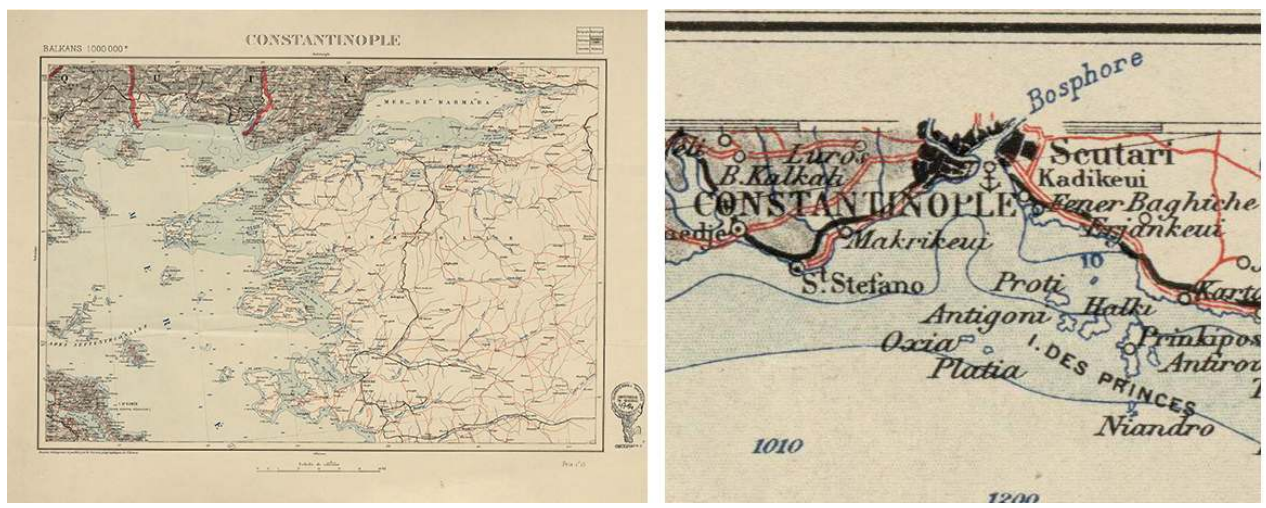

Sur la feuille Constantinople de la carte des Balkans à l'échelle un millionième, la ville considérée occupe seulement quelques millimètres de côté. Dans les catalogues classiques, elle apparaît tout d'abord à ce nom, au même titre que les plans de la ville. Constantinople, Paris, Service géographique de l'armée, [s.d., vers 1920].

(c) Cartothèque de l'université Paris 8 Saint-Denis.

En salle de lecture, cette carence des catalogues est en partie compensée par les informations apportées par les cartothécaires, par les tableaux d'assemblage, voire par les fichiers manuels. En ligne, le lecteur ne dispose pas d'autres renseignements que ceux du catalogue. Pour répondre à ses requêtes, le développement de nouveaux outils de recherche et de navigation par localisation géographique constitue le principal enjeu relatif à la mise à disposition des collections en ligne au cours des prochaines années. 
25 Encore peu d'établissements disposent d'un outil de recherche par localisation géographique. Le premier site web à proposer des tableaux d'assemblage interactifs en ligne a été développé par la Bibliothèque nationale d'Écosse (National Library of Scotland) en 2007. Ils étaient alors en marge du catalogue général, comme un complément d'information, et ne constituaient pas des outils de recherche stricto sensu mais une aide à la sélection des feuilles au sein de chaque série. Quelques années plus tard, le site web CartoMundi, dédié aux séries cartographiques, était organisé autour d'une interface de recherche par localisation géographique et des tableaux d'assemblage interactifs. En 2011, Map Warper, héritier d'un site plus ancien, proposait à la fois un service de géoréférencement en ligne, un outil de recherche géographique et une interface de visualisation des documents déposés par les utilisateurs de ce service. Pour les documents composés de plusieurs feuilles, ce site pallie l'absence de tableau d'assemblage par des mosaïques. Pour sa part, le site web Old Maps Online a été mis en service en 2013. Il s'agit d'un moteur de recherche géographique qui exploite les catalogues d'une vingtaine d'établissements. Il traite seulement des monographies dans la mesure où il ne permet pas de naviguer entre les multiples feuilles de chaque série ${ }^{21}$. Ensuite, d'autres établissements, plusieurs universités américaines en particulier, ont développé des outils plus rudimentaires de sélection des documents.

Suivant le mode d'organisation des interfaces de tous ces sites web, l'affichage des documents est considéré comme un aboutissement du processus de recherche. À l'issue de chaque consultation, le lecteur est invité à revenir à l'outil de recherche. Cette manière de procéder correspond effectivement à une transposition des usages des cartes analogiques qu'il est toujours difficile de confronter (dans certains établissements, les lecteurs ne sont pas autorisés à consulter plusieurs documents simultanément). Cependant, la publication de reproductions en ligne a pour effet de promouvoir l'émergence de nouvelles pratiques. En permettant les confrontations de manière systématique, elle ouvre la voie à une multiplication des consultations et des demandes de possibilités de « rebondir » à partir de chaque document, pour, en particulier, étudier l'évolution temporelle des lieux représentés. Or, une telle fonctionnalité n'est actuellement proposée par aucun site web alors que, grâce aux outils associés au géoréférencement, il est envisageable de développer assez facilement un système qui propose au lecteur d'autres figurations du lieu dont il consulte une carte. Sur cette base, l'interface pourrait aussi proposer de partager l'écran entre différentes reproductions pour en faciliter la confrontation.

\section{Pour conclure}

Nous l'avons vu, la reproduction numérique des documents patrimoniaux présente de multiples avantages, à la fois pour les établissements de conservation et pour les lecteurs. Pour les premiers, l'enjeu le plus important réside dans la possibilité d'inverser les étapes de traitement des documents. Cette inversion permettrait d'accélérer la rétro-conversion des catalogues dont le programme actuel s'échelonne sur des décennies et constituera assez rapidement un point de blocage pour la numérisation. Cependant, les cartothécaires résistent à s'engager dans un nouveau processus de catalogage parce que le format des catalogues actuellement en service exclut de prendre en charge les aspects géographiques de manière satisfaisante ${ }^{22}$. Pour exploiter toutes les capacités des moteurs de recherche géographique, il est nécessaire de compléter la description bibliographique de chaque 
document par une information qui rende compte du périmètre qu'il représente sur le globe terrestre. Or, les outils d'enregistrement de cette donnée restent à intégrer aux catalogues de la majorité des établissements. Par ailleurs, les informations géographiques ne sont pas directement disponibles sur les documents, il ne s'agit pas seulement de les lire et de les enregistrer mais il faut les construire et, pour au moins la moitié des documents, cette opération doit être effectuée par un spécialiste. Autrement dit, un catalogage performant des cartes doit passer par un renouvellement du métier de cartothécaire et par l'intégration de professionnels de la géomatique au sein des établissements ${ }^{23}$.

Enfin, pour les lecteurs, le renouvellement des pratiques de recherche, suggéré par les premières expériences, constitue de toute évidence l'aspect le plus prometteur du développement des reproductions numériques des documents cartographiques anciens. Il est cependant trop tôt pour en évaluer la portée.

\section{NOTES}

1. - CartoMundi est un site web documentaire dédié aux séries cartographiques. Il a été développé dans le cadre de la Maison méditerranéenne des sciences de l'homme à Aix-en-Provence, par l'université d'Aix-Marseille et le CNRS, avec la collaboration de l'Institut national de l'information géographique et forestière (IGN) et de la Bibliothèque nationale de France (BnF), avec le soutien de plusieurs collectivités territoriales et des laboratoires Telemme et Labex-MED. Voir le site : http://cartomundi.fr [consulté le 06/02/2018].

2. - ARNAUD, Jean-Luc. «Cataloguer/rechercher des cartes. Le référencement géographique en question ». Documentaliste-Sciences de l'information, vol. 51, n 3-2014, p. 69-79 et Id. «Organizing the Cartographic Series. New Technical and Conceptual Contexts ». Dans YAP, Joseph M., PEREZ, Martin Julius, AYSON, Maria Cecilia, ENTICO, Gladys Joy (dir.). Special Library Administration, Standardization and Technological Integration. Hershey: Information Science Reference, 2015, p. 267-304.

3. - Une série cartographique est une carte dont le rapport entre l'échelle de réduction, l'étendue du territoire représenté et le format du papier disponible impose son découpage en plusieurs feuilles.

4. - Pour les chercheurs qui s'intéressent à la gravure, aux techniques de dessin, aux filigranes... les reproductions numériques ne permettent pas de conduire les investigations nécessaires. Ils ont le plus souvent besoin de consulter les originaux.

5. - Ce qui est toujours le cas pour les documents numérisés qui ne sont pas libres de droits.

6. - Au cours des dernières années, plusieurs établissements ont procédé à la vente de leurs collections après les avoir reproduites.

7. - Le Groupe des cartothécaires de la Ligue des bibliothèques européennes de recherche (LIBER), a engagé ses premières réflexions en la matière au congrès d'Amsterdam en 2008 ; voir : MOORE, John. «The Future of Map Library and Map Librarian..., 1-5 July 2008 ». Liber Quarterly, 18, 2008, p. 227-237. Ensuite, les séances consacrées aux nouvelles technologies et à leurs usages ont été plus nombreuses à chaque congrès. Pour celui de 2010 (Tallinn), voir les sites: http:// www.nlib.ee/html/yritus/liber09/prog.html; pour celui de 2012 (Barcelone), http:// 
cartography.web.auth.gr/ICA-Heritage/MAGIC/Conferences/Barcelona_2012.pdf ; pour celui de $2014 \quad$ (Ljubljana), http://cartography.web.auth.gr/ICA-Heritage/MAGIC/ MAGIC_Letter_290514.htm; pour celui de 2016 (Riga), http://cartography.web.auth.gr/ICAHeritage/Riga2016/fil/proceedings.html. Pour sa part, la commission Digital Technologies in Cartographic Heritage, de l'Association cartographique internationale, a été fondée en 2007. Depuis 2012, elle est associée aux cartothécaires européens au sein du groupe MAGIC, voir le site: http://cartography.web.auth.gr/ICA-Heritage/MAGIC/ [consultés le 06/02/2108].

8. - Les formats de description proposés pour les rétroconversions sont si peu adaptés aux singularités de la cartographie que de nombreux cartothécaires ont préféré conserver des fichiers manuels. Ainsi, à la BnF, on estime à 350000 le nombre des documents cartographiques en série dont le catalogage n'est pas informatisé : SARAZIN, Jean-Yves. «CartoMundi, un nouveau mode d'accès au patrimoine cartographique ». Conférence SIG 2014, Versailles, 2 octobre 2014. Dans de nombreux autres établissements, chaque série est cataloguée à travers un seul bordereau qui ne précise pas le nombre de feuilles de la collection.

9. - REVELLI, Carlo. "L'assassinat des catalogueurs ». Bulletin des bibliothèques de France, 4, 2005, p. 13-19.

10. - Ce dispositif a été présenté pour la première fois en avril 2012 au congrès du groupe des cartothécaires de LIBER, à Barcelone : ARNAUD, Jean-Luc. « CartoTXT. Cataloguing the series in a semi-automatic way». En 2013, un projet de développement de ce dispositif - Automatiser le catalogage de la cartographie - porté par CartoMundi, a donné lieu à une association entre plusieurs laboratoires de l'université d'Aix-Marseille - USR 3125, UMR 7296 et UMR 7343. Il n'a pas obtenu le soutien nécessaire à la mise en place du système correspondant.

11. - Google Earth (à partir de 2005) ou encore Marble. Les documents sont alors enregistrés sous la forme de fichier KML (Keyhole Markup Language).

12. - ROSE, Rafael. « The map library after digitalization: new challenges ». Congrès Liber, groupe des cartothécaires, Tallinn, 18 juin 2010.

13. - Dans l'exemple de deux plans de Damas publiés au début des années 1920, la confrontation de la répartition géographique des erreurs de calage a permis d'établir leur filiation alors même que l'un est en arabe et l'autre en allemand: ARNAUD, Jean-Luc. "Corpus cartographique pour l'histoire de Damas, Syrie, à la fin de la période ottomane ». Imago Mundi, 2001, p. 46-70.

14. - Il existe plusieurs outils de géoréférencement disponibles gratuitement en ligne, dont: https://mapwarper.net/ ou encore https://www.georeferencer.com/ [consultés le 06/02/2018].

15. - Voir le site : https://www.geoportail.gouv.fr/. Ce site a été ouvert au public en juin 2006 [consulté le 06/02/2018].

16. - ARNAUD, Jean-Luc. La Carte de France, 1756-2018. Marseille : Parenthèses, à paraître.

17. - Ce document porte le titre "Carte au 1/50 000 de 1950 », son examen indique qu'il résulte de l'assemblage de feuilles très diverses. Si la plupart ont effectivement été publiées au cours des années 1950, leurs relevés s'échelonnent au cours des 25 années précédentes. Voir le site: https://remonterletemps.ign.fr/ [consulté le 06/02/2018].

18. - Jusqu'à la Première Guerre mondiale, la plupart des cartes commercialisées sous forme pliée étaient découpées en rectangles de taille régulière qui étaient ensuite collés sur toile avec des espacements qui en facilitaient le pliage.

19. - La liste des mosaïques compte 734 entrées mais les documents ne sont pas disponibles pour plus de la moitié. Voir le site : https://mapwarper.net/ [consulté le 06/02/2018].

20. - Norme Afnor Z44-067, la version actuellement en service date de septembre 1991.

21. - En proposant plus de 200000 reproductions en ligne, le site web de la Bibliothèque nationale d'Écosse (National Library of Scotland) est le plus important en la matière. Son responsable, Christopher Fleet, travaille depuis plus de dix ans à la numérisation des collections et à leur publication en ligne, voir le site : https://maps.nls.uk/. Pour sa part, l'outil de référencement géographique exploité par Old Maps Online - bounding box - est assez sommaire, il ne permet pas 
de rendre compte du périmètre de chaque document et retourne une multitude de réponses, pas toujours pertinentes, voir le site : http://www.oldmapsonline.org/ [consultés le 06/02/2018].

22. - En fait, la norme prévoit un champ géographique qui permet d'enregistrer les coordonnées des limites de chaque document. Cependant, les calculs nécessaires au renseignement de ce champ sont chronophages. Ils sont aussi à l'origine de multiples erreurs. Un test effectué dans le cadre de CartoMundi, à partir d'une extraction de la base de la BnF, a montré que ces données sont difficilement exploitables. Par ailleurs, ce champ n'enregistre pas le périmètre effectif de chaque feuille mais seulement son périmètre circonscrit de telle manière qu'il n'est pas utilisable pour les cartes en séries. Ce n'est pas un hasard s'il existe à notre connaissance un seul outil de recherche qui exploite ce champ, cela au prix d'une démultiplication des requêtes pour les lecteurs : ARNAUD, Jean-Luc. «Cataloguer / rechercher des cartes... », art. cit.

23. - Il n'est pas indifférent que les cartothèques universitaires américaines et canadiennes, qui ont développé des outils de recherche par localisation géographique, comptent depuis longtemps des géomaticiens au sein de leurs services.

\section{RÉSUMÉS}

Cette contribution consacrée aux reproductions numériques des documents cartographiques patrimoniaux est le fruit d'une double expérience: celle du montage et du pilotage du programme CartoMundi - Valorisation en ligne du patrimoine cartographique et celle de plusieurs années de travail en histoire de la cartographie et de l'iconographie. Les spécificités des documents cartographiques, par rapport aux textes, mobilisent des processus cognitifs particuliers qui déterminent un mode d'investigation singulier. On ne lit pas une carte, on la consulte; on ne la consulte pas a priori mais avec des questions. Dans ce contexte, cet article examine les enjeux portés par leur reproduction numérique et par les outils de recherche dans les catalogues. Il montre que les documents cartographiques ne peuvent pas être traités comme de simples images et que l'enregistrement de leur localisation géographique offre des opportunités encore peu exploitées qui mériteraient de plus amples développements.

This paper deals with the digital reproduction of map heritage. It is the result of a double experience: that of creation and management of the programme entitled CartoMundi - Online enhancement of the cartographic heritage and that of several years' work in the field of cartography and picture history. In relation to texts, the specificities of cartographic material mobilise particular cognitive processes that are at the origins of a particular mode of investigation. We do not actually read a map, we consult it. And, we do not consult a map a priori, we consult it in order to answer questions. This is the context in which this paper examines the issues raised by digital reproductions and research tools into the catalogues. It shows that cartographical material cannot be recorded as simple images. On the contrary, its treatment through geographic information systems offers still unexploited opportunities that deserve larger development. 


\section{INDEX}

Mots-clés : cartographie, carte, digitalisation, cartothéconomie, numérisation, série cartographique, CartoMundi, patrimoine, site web, information géographique, géoréférencement, catalogage, outil de recherche, outil de navigation

Keywords : cartography, map, carto-economy, map collection study, digitisation, cartographic series, CartoMundi, heritage, website, geographic information, geo-referencing, cataloguing, research tool, navigational tool

\section{AUTEUR}

\section{JEAN-LUC ARNAUD}

Directeur de recherches au CNRS, Aix Marseille univ, CNRS, laboratoire Telemme. MMSH Jlarnaud@mmsh.univ-aix.fr 